Proc. Indian Acad. Scí. (Earth Planet. Sci.), Vol. 89, Number 2, July 1980, pp. 121-132.

(C) Printed in India.

\title{
Cosmic ray effects in the solar system objects
}

\author{
$\mathrm{N}$ BHANDARI and $\mathrm{M} N$ RAO \\ Physical Research Laboratory, Ahmedabad 380 009, India
}

MS received 3 August 1979 ; revised 1 May 1980

\begin{abstract}
Long term characteristics of solar and galactic cosmic rays, as revealed by the study of their nuclear effects in lunar, meteoritic and terrestrial samples are summarised. The data so far available on radioisotopes, noble gases and tracks, though limited, are consistent with nearly constant fluxes and composition during different epochs over billions of years; one exception is ${ }^{14} \mathrm{C}$ activity in the earths atmosphere over the past few hundred years, suggesting a variation in the solar activity. Other small or brief variations, which cannot be ruled out as yet, require better estimation of depth and size dependence of nuclear effects in rocks before they can be attributed to cosmic rays.
\end{abstract}

Keywords. Cosmic rays; solar flares; meteorites; lunar samples.

\section{Introduction}

Astrophysical considerations lead us to believe that cosmic rays must have existed from the very beginning, at least since the initial formation of stars in our galaxy. The galactic cosmic ray (GCR) intensity is expected to depend on the nearby stellar population leading to local fluctuations from time to time. Chemical evolution of matter, as a result of its repeated cycling through the nucleosynthetic processes in the stellar interior may lead to gradual enrichment of heavy nuclei (Cameron 1970, 1973) and this would be reflected in the cosmic ray abundance pattern. In the interplanetary space, the cosmic rays from the sun (SCR) dominate at low energies (below about $100 \mathrm{MeV} / \mathrm{n}$ ). Solar cosmic rays also show energy and abundance variations over short periods, as observed in recent experiments on balloons and satellites, but little information exists on palaeoactivity of the sun.

Some of these aspects can be verified experimentally from a study of cosmic ray effects in solar system materials, like meteorites and lunar samples. A comparison with similar records on the earth enables us to distinguish the true interplanetary phenomenon from those of a local origin. Our knowledge of the prehistory of cosmic rays has steadily increased as the sequence of events in the evolution of solid objects in our solar system has become clearer. At different stages of the evolution of our solar system, the cosmic rays have left their vestigial records, which in many cases, with the advent of advanced techniques, have come

P. (A) $\rightarrow 1$ 
within the reach of our understanding. In this paper, we attempt to trace this sequence and summarise the information which has been obtained from the fossil cosmic ray records in meteorites, moon and the earth. Some of these results have been discussed earlier (Bhandari 1976).

\section{Cosmic ray effects}

Various components of cosmic radiation give rise to different types of records in rocks and these can be selectively studied. The most prominent effects caused by the nucleonic component of cosmic rays are the nuclear reactions and the radiation damage, both of which can be quantitatively estimated and related to the intensity and energy of the cosmic ray particles as well as their composition. For example, protons give rise to nuclear interactions with target material resulting in a large variety of stable and radioactive species. The major constituents of iron meteorites are iron and nickel and that of lunar samples and stone meteorites are $\mathrm{Si}, \mathrm{O}, \mathrm{Mg}, \mathrm{Al}, \mathrm{Fe}, \mathrm{Ni}, \mathrm{Ca}, \mathrm{Na}, \mathrm{Mn}$, etc and therefore a large number of stable as well as radioactive isotopes are produced in these interactions. The cosmogenic radioactive isotopes measured in meteorites range from ${ }^{52} \mathrm{Mn}$ (half-life 5.5 days) to ${ }^{40} \mathrm{~K}$ (half-life $1.2 \times 10^{9}$ years). Some of these are shown in figure 1 . The nuclear effects of neutrons and alpha particles can also be distinguished in some cases by their specific formation reactions. The rate of production $P_{1}$ of a particular isotope $i$,

$$
P_{i}(d)=\sum_{i} \int_{E_{T}}^{\infty} N_{j} \cdot \sigma_{i j}(E) \cdot I(E, d) d E
$$

depends on many factors like concentration of target elements $\left(N_{j}\right)$, shielding due to overlying material $(d)$, energy spectrum of nuclear active particles $I(E, d)$, cross-sections and their energy dependence $\sigma_{i}(E)$. The exposure history of the material, geometry of exposure, erosion and fragmentation in space therefore determine the rate of production. Furthermore, if $I(E)$ is time-dependent, these variations have to be taken into account. Solar cycle variations in $I(E)$ are well established but its long term function is not known and the approach has been to interpret any observed deviation from the expected value (for the presentday value of $I(E)$ ) in terms of time variations of cosmic rays. $\sigma_{i j}$ is well-known

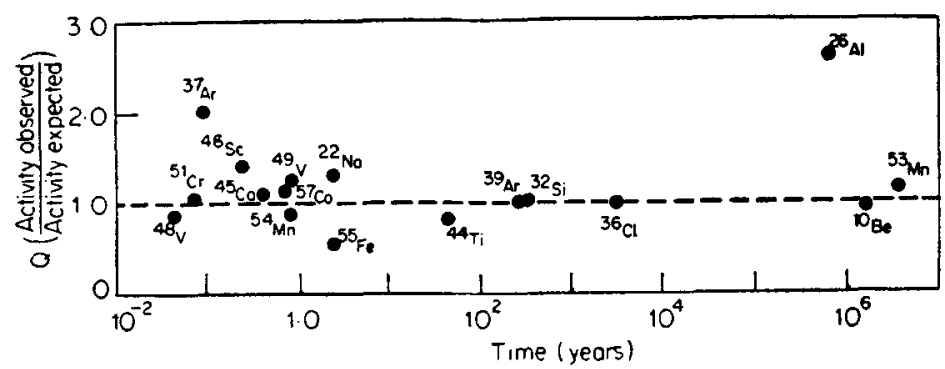

Figure 1. The ratio of observed to expected activity, $Q$, as a function of half life of isotope as observed in the meteorite Aroos (Arnold et al 1961). Points are identified by the radioisotopes. 
in case of several isotopes and Reedy and Arnold (1972) and Bhandari et al (1979) have described procedures for calculation of $I(E, d)$ and $P_{i}(d) . \quad I(E)$ decreases steeply with energy and energy spectra of various cosmic ray components, solar as well as galactic have been deduced (Reedy and Arnold 1972; Bhandari et al 1979; Lal 1974; Bhattacharya et al 1973). Experimentally, the radioactive species produced by cosmic rays are most easily distinguished. When the duration of exposure is larger compared to the isotope mean life, as is usually the case, the decay rate equals the rate of production of the isotope. The activity therefore gives the average flux of cosmic rays over the mean life of the isotope and therefore it is possible to evaluate time variations in average cosmic ray intensity by comparing activity for radioisotopes of different mean life. The cosmic ray contribution to stable isotopes has been evaluated successfully in the case of rare gas isotopes. This becomes possible because galactic and solar cosmic rays, interacting with lunar surface materials, produce spallation noble gas components having characteristic isotopic compositions. In favourable cases, it is possible to quantitatively resolve these spallation spectra to distinguish the SCR effects from those due to GCR in lunar rocks and fines (Bhai et al 1979).

Apart from these isotopic changes, an intense dose of cosmic ray particles results in radiation damage of crystalline structure of minerals present. in the rocks (Fleischer et al 1975). This can be seen very clearly, for example, as an amorphous layer on the exposed surface of grains and has been attributed to solar wind ions in case of lunar grains (Bibring et al 1972; Maurette and Price 1975). At moderate dose and somewhat higher energy ( $\mathrm{MeV} / \mathrm{n}$ range), e.g. in case of solar flare and galactic cosmic ray nuclei, the tracks formed by heavy ions $(Z>20)$ can be revealed in some rock minerals and various charge groups can be selectively studied from their characteristic track lengths (Lal 1969, 1972). Some of the conclusions based on these observations have been summarised earlier (Bhandari 1976).

\section{Solar effects in meteorites and lunar samples}

The earliest stage in the history of the solar system relates to formation of grains. To start with, the grains probably formed in the circumsolar space as has been observed in the case of several other stars (Woolf and Ney 1969; Forrest et al 1975). Meteorites do contain a variety of exotic grains. Some grains with peculiar isotopic abundances of $\mathrm{Mg}$ and $\mathrm{O}$, identified in the Allende meteorite (Clayton et al 1973; Gray and Compston 1974; Lee et al 1977) are thought to be pre- or extra-solar. These grains do not show any effects due to implantation of low energy interstellar ions ( $\mathrm{keV}-\mathrm{MeV}$ range e.g. due to interstellar wind) indicating that either they were shielded or got separated from the host cloud at high temperatures and were quickly embedded into a planetary debris without having a chance of getting irradiated. Individual grains in some other chondrites show records of independent irradiation by low energy cosmic rays as deduced from anomalously high track density gradients, too steep to be acquired at their observed location within the meteorite, as well as implanted solar wind ions (Lal and Rajan 1969; Pellas et al 1969). These records show near surface or unshielded irradiation. It has not been possible to ascertain the time of irradiation 
of these grains but it is plausible that these records go back to the periods, immediately following their formation, making them suitable for monitoring the early activity of the sun.

Apart from the track-rich grains in meteorites, which may have the oldest solar cosmic ray records, more recent records, dating back to about 1 b.y. have been found in lunar cores. Since the flux of heavy solar particles decreases steeply with energy (approximately as $E^{-3}$ at energies below about $30 \mathrm{MeV} / \mathrm{n}$, figure 2), the track production rate also decreases with depth within a grain. For a random exposure at various depths, as may occur in case of lunar soil under the effect of micrometeorite impacts, the track density acquired during shallow exposure therefore dominates; the effects of deeper exposure being usually negligible. Therefore different strata in the lunar soil, having been once exposed near the surface, mainly show the solar particle records. The largest core, e.g. the $2.5 \mathrm{~m}$ long Apollo 15 core, contains 54 major layers which appear to have been deposited sequentially over the last billion years (Bhandari et al 1973). Based on their analysis, it has been possible to reconstruct the solar particle history for various epoches going back to about 1 b.y. (Bhandari et al 1973). The lunar rocks have still shorter periods of exposure-of only a few million years. On this time scale, as a consequence of large cratering events, the lunar rocks undergo erosion, fragmentation and burial in the lunar soil. Thus for a period extending back to $10^{3}-10^{9}$ years, the lunar grains and rocks can be used as monitors of the solar activity.

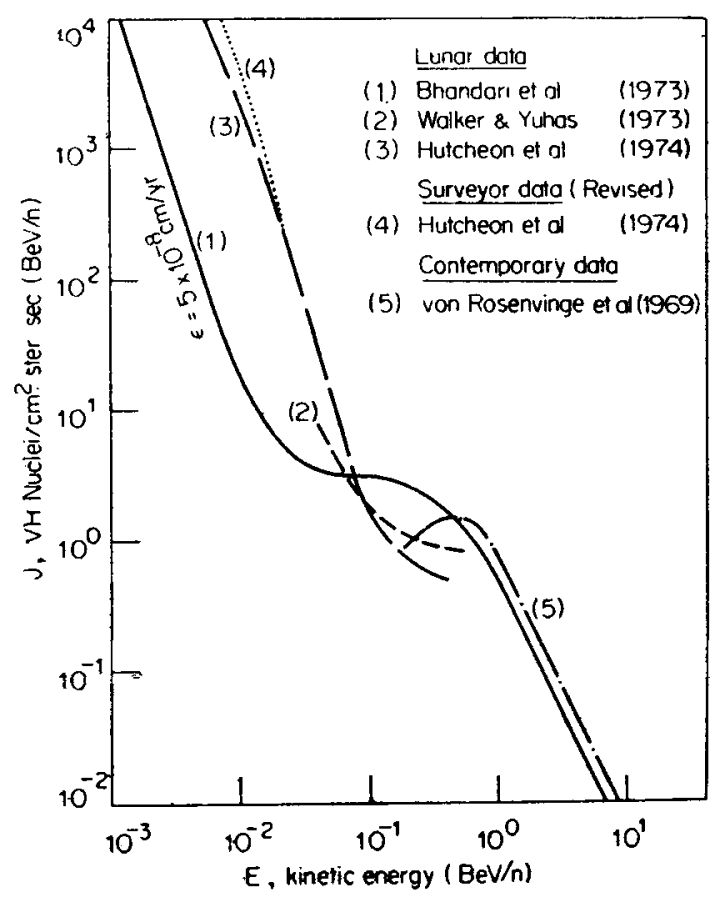

Figure 2. Energy spectrum of cosmic ray VH nuclei as deduced from a variety of studies. At low energies there is a large discrepancy, mainly because of the offects of erosion. 
Extensive studies of these samples have been made over the last few years for induced radioactivity, heavy nuclei tracks, noble gas systematics etc and the important conclusions of these studies can be briefly summarised as follows :

(i) Solar wind gases and solar flare tracks are found to exist in gas-track rich grains in meteorites and lunar cores, leading to the inference that the solar wind and flare activity have been present all the way back to several billion years approximately at the current level. Poupeau et al (1974) showed that the track rich meteoritic grains are also gas-rich and, in a bulk sample, the proportion of crystals with implanted solar wind is similar to the proportion of solar flare irradiated crystals. These results support an intimate association of solar flare tracks and implanted solar wind He. Bhattacharya et al (1975) from a correlation study of solar wind ${ }^{36} \mathrm{Ar}$ and track-rich grains from gas-rich meteorites and lunar (oils inferred that the ratio of average solar wind to solar flare (very heavy) particles $\mathrm{VH}>0.5 \mathrm{MeV} / \mathrm{n})$ was much less $(\sim 1 / 100$ or so) when meteoritic grains were irradiated on the surface of a parent body, compared to the values at 1 A.U. for the last 1-2 b.y. corresponding to the lunar sample records. This correlation is shown in figure 3 .

(ii) The shape of the energy spectrum of solar VH nuclei (defined by the exponent $\gamma=3 \pm 0.3$ in $\mathrm{dN}=E^{-\gamma} d E$ ) in the past has been similar to that observed in contemporary cosmic rays at least upto about $30 \mathrm{MeV} / \mathrm{n}$ (Lal 1974; Lal and Rajan 1969) although it has not been possible to deduce the actual fluxes. This conclusion is supported by the recent work in the Murchison chondrite which is consistent with the surveyor glass data (Goswami et al 1980).

(iii) In addition, the gross composition VVH/VH in solar flares seems to be as high as $5 \times 10^{-2}$ in Murchison (Goswami et al 1980), significantly higher than the cosmic abundance ratio found in galactic cosmic rays (table 1). The results

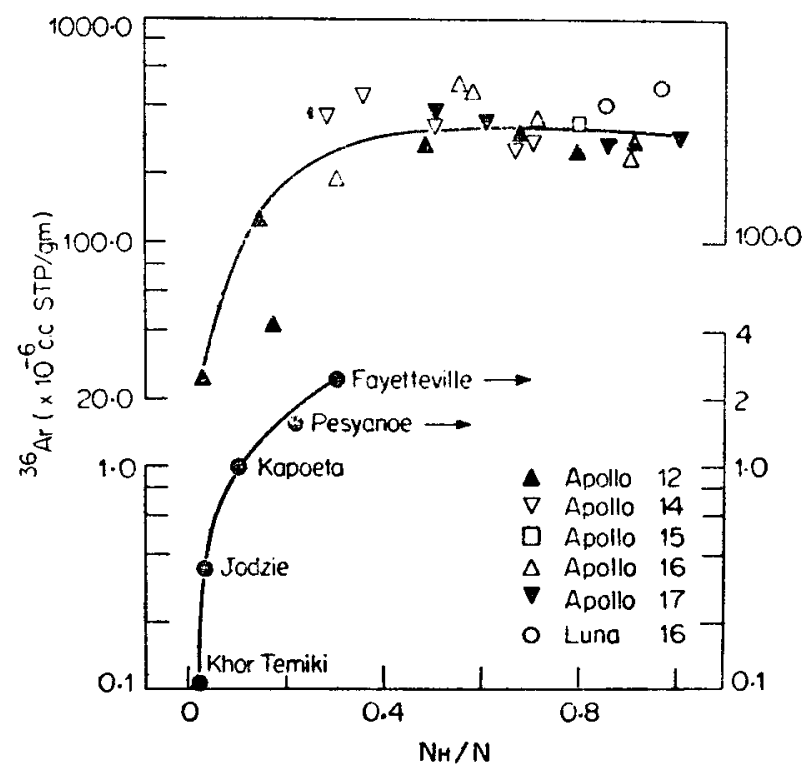

Figure 3. A comparison of ${ }^{36} \mathrm{Ar}$ concentrations vs $\mathrm{NH} / \mathrm{N}$ values for lunar soil and dark portions of gas-rich meteorites (Bhattacharya et al 1975). 
Table 1. Abundance ratio (VVH/VH) in various sources.

\begin{tabular}{|c|c|c|}
\hline Source & $\begin{array}{l}\mathrm{VVH} / \mathrm{VH} \\
\left(10^{-3}\right)\end{array}$ & $R \neq f e r e n c e s$ \\
\hline $\begin{array}{l}\text { Solar photosphere } \\
\text { Recent solar flares } 1\end{array}$ & $1 \cdot 2-1 \cdot 4$ & Hauge and Engvold (1970) \\
\hline 1-2 MeV/n & 50 & Goswami and Lal (1975) \\
\hline$\approx 5 \mathrm{MeV} / \mathrm{n}$ & 13 & Bhandari et al (1973) \\
\hline $\begin{array}{c}>10 \mathrm{MeV} / \mathrm{n} \\
\text { Ancient solar flares }\end{array}$ & 2 & Bhandari et al (1973) \\
\hline$>10 \mathrm{MeV} / \mathrm{n}$ & 50 & Goswami and Lal (1975) \\
\hline Solar system & $2 \cdot 1$ & Cameron (1968) \\
\hline Galactic cosmic rays & & \\
\hline $\begin{array}{l}<50 \mathrm{MeV} / \mathrm{n} \\
\sim 50 \mathrm{MeV} / \mathrm{n}\end{array}$ & $\begin{array}{l}1 \cdot 6 \\
2 \cdot 3\end{array}$ & $\begin{array}{l}\text { Bhandari et al (1973) } \\
\text { Br.andari et al (1973) }\end{array}$ \\
\hline$>100 \mathrm{MeV} / \mathrm{n}$ & $1 \cdot 3 \pm 0 \cdot 6$ & Bhandari and Padia (1974) \\
\hline
\end{tabular}

(1) These refer to the past few million years covered by lunar rock exposure time.

(2) These refer to a few billion years ago corresponding to irradiation of track rich meteorite grains.

also indicate that the acceleration mechanism from the sun has not changed much over the past (Bhandari et al 1973).

(iv) The average energy of solar wind ions has been about $1 \mathrm{keV}$ as inferred from the thickness of the radiation damaged regions in lunar soil grains (Maurette and Price 1975) irradiated at different times.

(v) The radioisotope ${ }^{26} \mathrm{Al}$ observed at the surface of different lunar rocks exposed for different spans of time is found close to the expected levels leading to the conclusion that the average solar flare activity has remained at the presen $t$ level (Bhandari et al 1976) for the last 2 million years. These data are shown in figure 4 where the ratio of observed to expected activity is plotted as a function of time. Recent results of Kohl et al (1978) confirm this conclusion of near constancy of cosmic rays. The solar proton spectrum deduced by Bhandari et al (1976) is best represented by $d N=K e^{-R / 150} d R$ with ( $4 \pi$ omnidirectional) flux $J(>10 \mathrm{MeV} / \mathrm{n})=140 \pm 10 \mathrm{p} \cdot \mathrm{cm}^{-2} \mathrm{sec}^{-1}$. These data are shown in figure 5 where the calculated depth profile of ${ }^{26} \mathrm{Al}$ with various parameters $J$, Ro and erosion rate is also shown for comparison. The data of Kohl et al (1978) indicate much lower fluxes. A part of this discrepancy may be due to the difficulty in independently determining the solar exposure ages of the rocks. Nonetheless the near constancy of solar flare fluxes are supported by the observations of both the groups (Bhandari et al 1976; Kohl et al 1978).

\section{Galactic cosmic ray records in meteorites and lunar samples}

Since in the interplanetary space the galactic cosmic rays dominate at energies $\geqslant 100 \mathrm{meV} / \mathrm{n}$. Similar studies carried out in the lunar rocks, cores and meteorites at high energy have been related to characteristics of galactic cosmic rays. Production profiles of radionuclides and rare gases were not known with good 


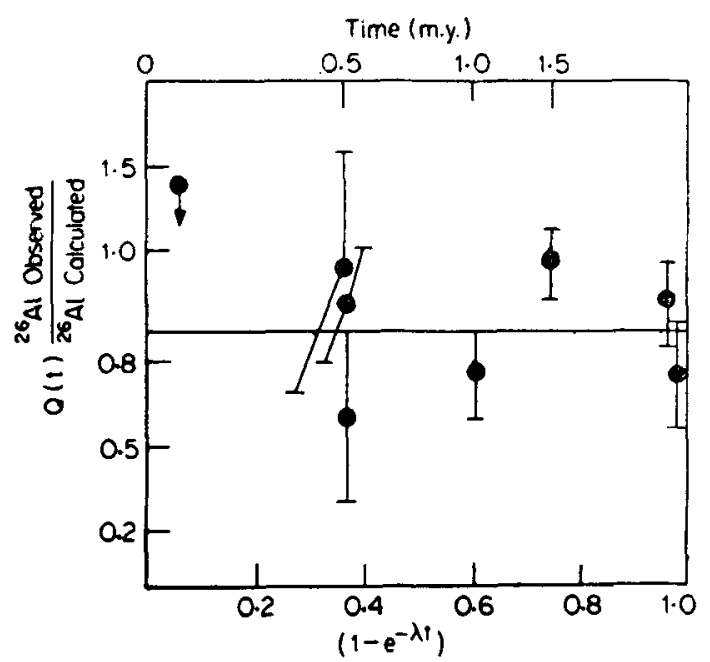

Figure 4. The ratio of observed to expected activity $Q$ of ${ }^{26} \mathrm{Al}$ as a function of exposure time of the rocks. The points are identified by the rock numbers (Bhandari et al 1976).

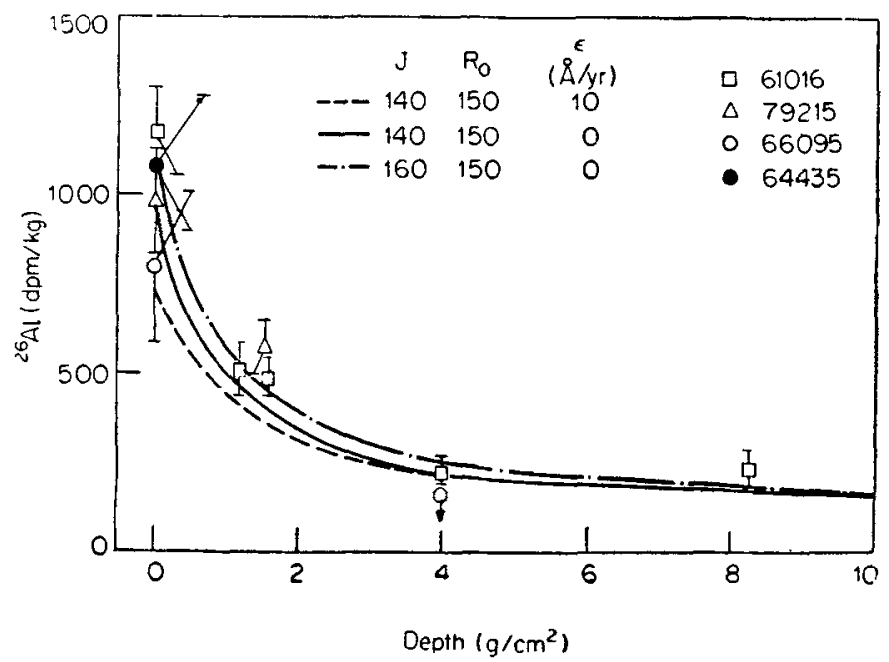

Figure 5. Observed activity of ${ }^{26} \mathrm{Al}$ (corrected for growth corresponding to exposure time of rocks) as a function of depth in various rocks. The solid lines indicate calculated profiles for rock 61016 for different values of $J$ with erosion rate $10 \mathrm{~A} / \mathrm{yr}$ and without erosion (Bhandari et al 1976).

enough accuracy so far and therefore quantitative inferences were not so reliable. However recent studies (Bhandari et al 1979; Reedy et al 1979) give the depth profile of some stable and radio-isotopes in meteorites of different sizes. The iron meteorites have exposure ages of the order of a billion years whereas exposure ages of stone meteorites cluster around tens $(<50)$ of million years (Begemann 1971), thus making them suitable for evaluation of long-term variation of cosmic rays. A comparison of the lunar and meteorite data also allow estimation of the spatial variations in the interplanetary space. The following are some important conclusions drawn from studies carried out so far. 
(i) The observed activity of different radioisotopes with half life ranging from 5 days to about a billion years is in close agreement with the expected production based on a single energy spectrum leading to the conclusion of near constancy of cosmic ray fluxes in the past as first proposed by Arnold et al (1961). The data on which this conclusion was based are shown in figure 1 where the ratio $Q$ of the observed to expected activity for many isotopes in Aroos iron meteorite is shown as a function of their half life. Recently Schaeffer (1975) has discussed the $\mathrm{Ar}^{39}-\mathrm{Cl}^{36}-\mathrm{K}^{40}$ data and inferred temporal variations over a period of $4 \times 10^{2}-4 \times 10^{5}-10^{9}$ years which are attributed to local cosmic ray variation in the circumsolar space. Since the orbital plane of the sun is inclined to the principal galactic plane, these observations have been related to spatial variation across the galactic plane. These limited observations suggest the possibility of a $50 \%$ reduction in intensity at a distance of $70 \mathrm{pc}$ away from the galactic plane, similar to the distribution of matter in the galaxy.

(ii) By comparing the radioactivity of ${ }^{26} \mathrm{Al}$ produced in lunar samples and stone meteorites (after correcting for target chemistry etc.), it was found to be close to the expected activity based on the present-day energy spectrum of cosmic rays (Bhattacharya and Bhandari 1976). The ratio of observed to expected activity varies from 0.82 for meteorites to 0.71 for the lunar samples thus leading to the conclusion that spatial variation of galactic cosmic rays (above a few hundred $\mathrm{MeV} / \mathrm{n}$ ) has been less than $+10 \% / A . U$. between 1 and about 3 A.U. in the interplanetary space. These results agree with recent observations of pioneer spacecraft.

(iii) A study of radionuclides of different half-lives produced in the Dhajala chondrite, which had an inclined orbit to the ecliptic, and fell at the time of solar minimum has provided heliolatitude variation in solar modulation to be estimated (Bhandari et al 1978). The data lead to $33 \pm 7 \%$ higher GCR proton fluxes $(~ \gtrsim 100 \mathrm{MeV})$ at heliolatitudes $>15^{\circ}$ during solar minimum.

(iv) The relative abundance of heavy ions $\mathrm{VVH} / \mathrm{VH},(Z \geqslant 30 / Z>26)$ has also been inferred by the track length distribution in lunar core grains, lunar rocks and irradiated grains in meteorites (Bhandari and Padia 1974). The results show small ( $\sim$ factor of 2$)$ variation over a billion year period. The ratio at the extreme range from 0.8 to $1 \cdot 3\left(\times 10^{-3}\right)$ which is within the expected experimental variations as shown in figure 6 .

\section{Cosmic ray records on the earth}

Radioisotopes produced in cosmic ray interactions with the air have been studied in atmosphere, precipitation, oceans and the deep sea sediments. Some terrestrial records refer to differential time windows, and thus offer an advantage of higher sensitivity and well-documented chronology as compared to meteorites and lunar sample studies. The most relevant measurements from the point of view of the long term characteristics of cosmic rays are that of ${ }^{14} \mathrm{C}$ in tree rings, and of ${ }^{10} \mathrm{Be}$ in deep sea sediments. The ${ }^{14} \mathrm{C}$ data in dated tree rings and recent samples show significant variations which have been attributed to Suess effect (depletion in ${ }^{14} \mathrm{C}$ activity after about $1900 \mathrm{AD}$, due to fossil fuel burning), nuclear tests (after $1950 \mathrm{AD}$ ) and de Vries effect (variation in ${ }^{14} \mathrm{C}$ activity due to climatic 


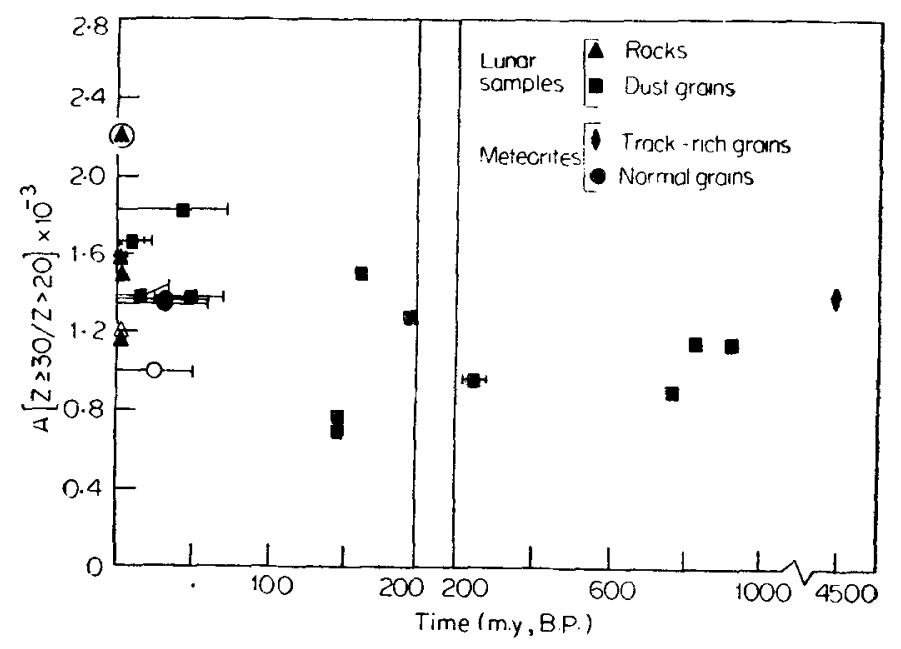

Figure 6. The flux ratio of $30 \leqslant Z<40$ relative to $20<Z<26$ nuclei in cosmic rays for different epochs in the past. The bar on each point represents the time span over which the sample was irradiated with galactic cosmic rays. The typical error in each ratio is $\pm 30 \%$. The open points represent data at high energy $(E=0.2$ to $2 \mathrm{beV} / \mathrm{n}$ ) and the solid symbols represent an energy range of 0.02 to $0.4 \mathrm{beV} / \mathrm{n}$. The circled triangle shows the marginal increase around $0.5 \mathrm{beV} / \mathrm{n}$ observed in rock 12020 (Bhandari and Padia 1974).

effect or change in production etc.). These have been reviewed recently (Damon et al 1978). The data show two minima and maxima and other minor variations between $\mathrm{AD} 1500$ and 1850 due to de Vries effect. The ${ }^{14} \mathrm{C}$ maxima have been attributed to increased production due to galactic cosmic rays during the Maunder and Sporer minima in solar activity (Eddy 1976). The ${ }^{14} \mathrm{C}$ activity sunspots and terrestrial climate seem to be correlated (Damon et al 1978). However a very careful work by Hays et al (1976) on deep sea cores indicate oxygen isotopic variations which can best be explained by the Milankovitch theory, i.e. due to the variation in solar energy received because of the orbital motion of the earth. Clearly if solar variability exists, it will be much more dominant and the Milankovitch effect will only be secondary. Kondrateyev and Nikolsky (1970) have suggested that solar constant and Wolf sunspot number $R$ are related. Also, the solar activity and GCR fluxes are anti-correlated. The variability of the solar activity can, therefore, be verified by studying GCR effects in meteorites. Forman et al (1977) have advanced arguments on the basis of ${ }^{39} \mathrm{Ar}$ data in meteorites to support the Maunder Minimum. Bhandari et al (1979) have now studied ${ }^{39} \mathrm{Ar}$ in meteorites which fell during 18th and 19th century. The effect of Maunder Minimum is very small, resulting in about $5 \%$ increase in ${ }^{39} \mathrm{Ar}$ activity. Figure 7 shows the preliminary results of this work. As a result of accurate estimation of depth effects in ${ }^{39} \mathrm{Ar}$ production, this work rules out any major $(10 \%)$ increase in GCR fluxes during the past few centuries (since about 1400 AD) although evaluation of the Maunder Minimum effects require more precise analysis which is in progress. ${ }^{10} \mathrm{Be}$ has been measured in detail (Somayajulu 1977; Inoue and Tanaka 1979) in dated deep sea cores upto about $2.5 \mathrm{~m} . \mathrm{y}$. in the past. These 


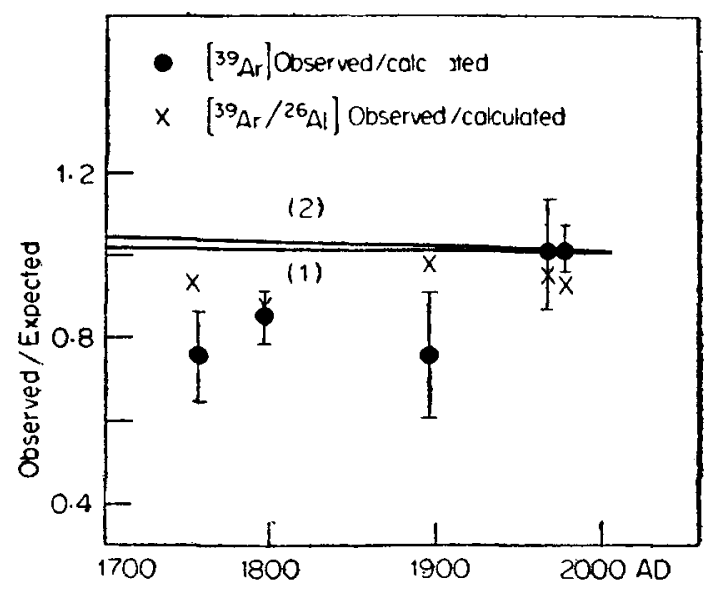

Figure 7. The ratio of observed $\left({ }^{39} \mathrm{Ar} /{ }^{26} \mathrm{Al}\right)$ to that expected as a function of the time of fall of the meteorite. The solid curves give the expected variation due to Maunder and Sporer minima (Bhandari et al 1979).

measurements also do not show any measurable variation of GCR fluxes ove this time period. There are some variations in the absolute ${ }^{10} \mathrm{Be}$ activity, but climatic, geochemical and environmental effects, which are not well-known, have to be taken into account before they can be ascribed to different cosmic ray fluxes. Inoue and Tanaka (1979) conclude that the variations, if any, do not exceed $\pm 10 \%$ for $2-7 \times 10^{5} \mathrm{yr}$. and $\pm 40 \%$ for $\sim 10^{5} \mathrm{yr}$. during the past $2.5 \mathrm{~m} . \mathrm{y}$. If any such GCR variation is caused by solar activity variations and consequent modulation, such an effect is rather small as already mentioned earlier in context with the measurement of ${ }^{28} \mathrm{Al}$ in lunar rocks.

\section{Application of cosmic ray records in solar system astrophysics}

With the large variety of records available in solar system materials, as discussed above, one can, in addition to learning about the pre-history of cosmic rays, use cosmic ray effects to reconstruct the events in the solar system and deduce insight into several astrophysical phenomena. A case in point is the detection of irradiated grains in the meteorites (Lal and Rajan 1969; Pellas et al 1969) which indicates one important process in the evolution of minor objects in the solar system. Recent results of Goswami and Lal (1979) suggest that the irradiation took place in cm-meter size bodies. Similarly the exposure ages of meteorites, lunar rocks and cores, processes connected with movement and deposition of soil on the moon and erosion in space have been evaluated using cosmic ray effects (Begemann 1971; Arrhenius et al 1971; Bhandari et al 1971).

We only refer here to some recent information about the processes operating in the sun which has resulted from study of lunar samples. Fireman (1976) has found evidence of ${ }^{14} \mathrm{C}$ produced by nuclear interactions of solar flare particles in the solar atmosphere, subsequently transported by solar wind and implanted in the surface of lunar soil grains. The observed ${ }^{14} \mathrm{C} / \mathrm{H}$ ratio is about $3 \times 10^{-11}$. 
The search for ${ }^{3} \mathrm{H}$ produced by the same mechanism has been negative, indicating a residence time in the sun much larger than the tritium mean life ( $\sim 50$ years). This is the first observation of a radioisotope in the solar wind and when extended to other radioactivities, would provide important information on time scales of processes occurring in the solar atmosphere.

Another important observation relates to the acceleration of solar flare ions in the sun. Price et al (1971) found relative enrichment at low energies of heavy ions like Fe over their photospheric abundance. Similar energy-dependent enhancement of ions with atomic number about 30 over $\mathrm{Fe}$ was observed by Bhandari et al (1973) in lunar soil grains and Goswami et al (1980) in track-rich grains in meteorites. Some of these results are summarised in table 1. These observations suggest that the acceleration mechanism of particles from the sun has been similar throughout the past. Goswami and Lal (1975) have also shown that the chargedependent enhancement does not continue all the way to low energies but the $\mathrm{VVH} / \mathrm{VH}$ ratio stabilises at about $2 \mathrm{MeV} / \mathrm{n}$. These observations are extremely valuable in understanding the acceleration and escape mechanisms of solar flare ions, although no satisfactory models exist so far.

\section{Conclusions}

In conclusion it can be stated that a variety of information about cosmic rays in the past has emerged from studies of terrestrial and extraterrestrial samples. Briefly, the intensity of solar particles has been roughly the same as now for at least a billion years from $\mathrm{keV}$ to $\mathrm{MeV}$ range, i.e. for solar wind as well as solar flare particles. The group composition has also not varied significantly over the past either. The composition of solar flare particles has not been similar to that of the solar photosphere, but there is a complex enrichment of heavier ions at lower energy. There is no conclusive evidence so far for an early active sun but with precise work on early condensates in meteorites, it may be possible to ascertain this point. For the galactic cosmic rays, the data so far available show little variation in intensity as well as composition over millions or even billions of years. Unfortuntaely most of these data are qualitative in nature but with the recent developments in nuclear techniques and availability of well documented extraterrestrial samples, a systematic work should lead to a better time and space resolution of cosmic ray effects with the required precision.

\section{Acknowledgements}

We thank Dr J N Goswami for useful discussions.

\section{References}

Arnold J R, Honda M and Lal D 1961 J. Geophys. Res. 663519

Arrhenius G, Liang S, MacDougall J D, Wilkening, L, Bhandari N, Bhat S, Lal D, Rajagopalan G, Tamhane A S and Venkatavaradan V S 1971 Proc. II Lunar Sci. p. 2583

Bhai N B, Gopalan K, Goswami J N, Rao M N and Venkatesan T R 1978 Proc. Lunar Planet. Sci. Conf. 9th 1629 (Pergamon)

Begemann F 1971 On the origin of the solar system Nice Symposium p. 250

Bibring J P, Duraud J P, Burrieu L, Jouret C, Maurette M and Meunier R 1972 Science 157 753 
Bhandari N 1976 Proc. Symp. Solar Planet. Phys. Physical Research Laboratory, Ahmedabad 171

Bhandari N, Bhattacharya S K and Potdar M B 1979 Lunar Planet. Sci. 10107

Bhandari N, Goswami J N and Lal D 1973 Proc. Lunar Sci. Conf. 4th Houston, USA (Pergamon) p. 2275

Bhandari N, Goswami J N, Lal D and Tamhane A S 1973 Astrophys. J. 185975

Bhandari N, Bhattacharya S K and Padia J T 1976 Proc. VII Lunar Sci. Conf. Houston, USA (Pergamon) p. 513

Bhandari N, Bhattacharya S K and Somayajulu B L K 1978 Earth Planet. Sci. Lett. 40194 Bhandari N and Padia J T 1974 Science 1851043

Bhandari N, Prabhakara H R and Raman T 1979 Lunar Planet Sci. 10110

Bhandari N, Bhat S G, Lal D, Rajagopalan G, Tamhane A S and Venkatavaradan V S 1971 Geochim. Cosmochim. Acta 32611

Bhattacharya S K, Goswami J N and Lal D 1973 J. Geophys. Res. 788356

Bhattacharya S K, Goswami J N, Lal D, Patel P P and Rao M N 1975 Proc. VI Lunar Sci. Conf. p. 3509

Bhattacharya S K and Bhandari N 1976 Proc. Symp. Sol. Planet. Phys. Physical Research Laboratory, Ahmedabad 261

Cameron A G W 1970 Space Sci. Rev. 15121

Cameron A G W 1973 Icarus 18407

Cameron A G W 1968 in The origin and distribution of the elements ed. L H Ahrens (New York : Pergamon)

Clayton R N, Grossman L and Mayeda T L 1973 Science 182485

Damon P, Lerman J C and Long A 1978 Annu. Rev. Earth Planet. Sci. p. 458

Eddy J A 1976 Science 1921189

Fireman E L 1976 Earth Planet Sci. Lett. 32185

Fleischer F L, Price P B and Walker R M 1975 Nuclear tracks in solids (Berkeley: University of California Press)

Forman M A, Schaeffer D A and Schaeffer G A 1977 Proc. XV Cosmic Ray Conf. Plovdiv p. 187

Forrest W J, Gillett F C and Stein W A 1975 Astrophys. J. 195423

Gray C M and Compston W 1974 Nature (London) 251495

Goswami J N, Lal D and MacDougall J D 1980 Proc. 16th Int. Conf. Cosmic rays (in preparation)

Goswami J N and Lal D 1979 preprint

Goswami J N and Lal D 1975 Proc. V Lunar Sci. Conf. Houston USA (Pergamon) p. 2643

Hauge $O$ and Engvold $O 1970$ Inst. Theoret. Astrophys. 0510 Rep. 31

Hays J D, Imbrie J and Shackleton N J 1976 Science 1941121

Inoue T and Tanaka S 1979 Nature (London) 277209

Kohl C P, Murrell M T, Russ G R III and Arnold J R 1978 Proc. IX Lunar Planet. Sci. Conf. 22299

Kondrateyev K Ya and Nikolsky G A 1970 Q. J.R. Meteorol. Soc. 569

Lal D 1974 Philos. Trans. R. Soc. London A277 395

Lal D 1969 Space Sci. Rev. 9623

Lal D 1972 Space Sci. Rev. 143

Lal D and Rajan R S 1969 Nature (London) 223 5203, 269

Lee T, Papanastassiou D A and Wasserburg G J 1977 Astrophys. J. 211 L 107

Maurette M and Price P B 1975 Science 187121

Pellas P, Poupeau G, Lorin J C, Reeves H and Audouze J 1969 Nature (London) 223272

Poupeau G, Kirsten T, Steinbrunn F and Storzer D 1974 Earth Planet. Sci. Lett. 24229

Price P B, Hutcheon I A, Cowsik R and Barber D J 1971 Phys. Rev. Lett. 26916

Reedy R C and Arnold J R 1972 J. Geophys. Res. 77537

Reedy R C, Herzog G F and Jessberger E K 1979 LA-UR-78-3220, Los Alamos Scientific Laboratory (preprint)

Schaeffer O A 1975 Proc. XIV Cosmic ray conf. (Munich) 113508

Somayajulu B L K 1977 Geochim. Cosmochim. Acta 41909

Woolf N J and Ney E P 1969 Astrophys. J. 155 L 181 